\section{Robust Adaptive Motion/Force Tracking Control of Uncertain Nonholonomic Mechanical Systems}

\author{
Masahiro Oya, Chun-Yi Su, and Ryozo Katoh
}

\begin{abstract}
The position/force tracking control of Lagrangian mechanical systems with classical nonholonomic constraints is addressed in this paper. The main feature of this paper is that 1) control strategy is developed at the dynamic level and can deal with model uncertainties in the mechanical systems; 2) the proposed control law ensures the desired trajectory tracking of the configuration state of the closed-loop system; 3) the tracking error of constraint force is bounded with a controllable bound; and 4) a global asymptotic stability result is obtained in the Lyapunov sense. A detailed numerical example is presented to illustrate the developed method.
\end{abstract}

Index Terms-Adaptive control, mechanical systems, motion/force control, nonholonomic constraints, robust control, tracking control, uncertainties.

\section{INTRODUCTION}

Nonholonomic constraints arise when there is a rolling or sliding contact in mechanical systems [16]. A large class of systems, such as wheeled vehicles, mobile robots, multifingered robotic hands, space robots, etc., involve nonholonomic constraints. Control of nonholonomic mechanical systems has attracted significant attention recently due to the demand for control of the above-referred systems [2]. Considerable efforts have been expended in designing stabilizing controllers, and research results can generally be classified in two classes. The first is kinematic control [2], [4], [17], which provides the solutions only on a pure kinematic level, yielding kinematic control such as driving speed. The second is dynamic control [3]-[13], taking inertia and forces into account and yielding physical controls such as driving torques. The interested reader may refer to the survey paper [2] for various control methods.

The tracking problem (tracking a reference trajectory), as a much more interesting issue in practice, has received relatively less attention in the literature. Depending on whether the nonholonomic systems are presented at a kinematic or dynamic level, the tracking problem can also be classified as either a kinematic tracking problem or a dynamic tracking problem. Similar to the stabilization case, most of the work reported to date on the tracking problem is at kinematic level (see [21] for references). However, it is evident that practical applications call for solutions at the dynamic level. Recognizing the importance of addressing the tracking control problem at the dynamic level, some effort has been devoted to this problem [10], [14], [15].

It is also important to note that constraints usually result from contact interaction of the machine and the environment. Therefore, the control of the forces of interactions is at least as important as the position control. Applying a conventional technique, one can reduce the number of state variables with those which provide motion complying with con-

Manuscript received November 2, 2001; revised February 27, 2002. This paper was recommended for publication by Associate Editor H. Arai and Editor I. Walker upon evaluation of the reviewers' comments.

M. Oya is with the Department of Control Engineering, Kyushu Institute of Technology, Tobata, Kitakyushu, 804-8550, Japan (e-mail oya@cntl.kyutech.ac.jp).

C.-Y. Su is with the Department of Mechanical and Industrial Engineering, Concordia University, Montreal, QC, H3G 1M8, Canada (e-mail cysu@me.concordia.ca).

R. Katoh, deceased, was with the Department of Control Engineering, Kyushu Institute of Technology, Tobata, Kitakyushu, 804-8550, Japan.

Digital Object Identifier 10.1109/TRA.2002.807528 straints. Physically, this implies that the constraints provide the necessary reactions. However, the magnitude of the constrained forces may not be desirable, as a result of control efforts on position errors. Whatever sophisticated control algorithms there may be, it is impractical unless the position and the force of interaction are controlled simultaneously. However, the literature on the force control of nonholonomic mechanical systems is sparse [10], [19], [20].

In this paper, we are concerned with Lagrangian mechanical systems with classic nonholonomic constraints. The challenge addressed here is to ensure, at the dynamic control level, desired trajectory and constraint force tracking in the presence of parameter modeling uncertainties. By assuming complete knowledge of the constraint manifold, and taking the internal dynamics of the systems into account, a full dynamics description suitable for motion and force control is derived by decomposing the system into two cascaded subsystems: a kinematic system and a new dynamic description. A robust motion/force tracking control algorithm is then derived, ensuring that the desired trajectory and the desired constraint force can be tracked. Stability analysis shows the desired position tracking and force tracking errors are bounded with a controllable bound. The application of the developed design procedure is illustrated by an example.

This paper is organized as follows. Methods developed in [10] for decomposing nonholonomic mechanical systems into two cascaded subsystems, a kinematic system and a lower dimensional dynamic system, is reviewed in Section II, and Section III presents a control strategy. The stability analysis for the complete system is also conducted in this section. Section IV provides illustrative examples using the proposed approach. In Section V, some conclusions are presented.

\section{Model of MechanicAl SyStem With NONHOLONOMIC CONSTRAINTS}

In this section, we are concerned with mechanical systems with a classical nonholonomic constraint, whose configuration space is an $n$-dimensional, simply connected manifold $\Re$, and whose dynamics are described, in local coordinates (termed generalized coordinates), by so called Euler-Lagrangian formulation as [5], [16]

$$
\begin{aligned}
D(\mathbf{q}) \ddot{\mathbf{q}}+C(\mathbf{q}, \dot{\mathbf{q}}) \dot{\mathbf{q}}+G(\mathbf{q}) & =J^{T}(\mathbf{q}) \lambda+B(\mathbf{q}) \tau \\
J(\mathbf{q}) \dot{\mathbf{q}} & =0
\end{aligned}
$$

where $\mathbf{q}$ denotes the $n$ vector of generalized coordinates; $\tau$ denotes the $r$ vector of generalized control input force; $\lambda \in R^{m}$ is the associated Lagrangian multipliers which expresses the contact force on the contact point between the rigid body and the environmental surface; $D(\mathbf{q})$ is the $(n \times n)$ symmetric, bounded, positive definite inertia matrix; $C(\mathbf{q}, \dot{\mathbf{q}}) \dot{\mathbf{q}}$ presents the $n$ vector of centripetal and Coriolis torques; $G(\mathbf{q})$ is the $n$ vector of gravitational torques; $B(\mathbf{q})$ is an $(n \times r)$ input transformation matrix, and is assumed to be known because it is a function of fixed geometry of the system; and $J(\mathbf{q})$ is the $(m \times n)$ constraint matrix. In the system (1) and (2), the constraint equation (2) is assumed to be completely nonholonomic for all $\mathbf{q} \in \Re^{n}$ and $t \in \Re$. To completely actuate the nonholonomic system, $B(\mathbf{q})$ is assumed to be a full-rank matrix and $r \geq n-m$.

Two simplifying properties should be noted about this dynamic structure.

Property 1: There exists a so-called inertial parameter $p$ vector $\beta_{p}$ with components depending on mechanical parameters (masses, moments of inertia, etc.,) such that [10]

$$
D(\mathbf{q}) \dot{\mathbf{v}}+C(\mathbf{q}, \dot{\mathbf{q}}) \mathbf{v}+G(\mathbf{q})=\Phi(\mathbf{q}, \dot{\mathbf{q}}, \mathbf{v}, \dot{\mathbf{v}}) \beta_{p}
$$


where $\Phi$ is a $n \times l$ matrix of known functions of $\mathbf{q}, \dot{\mathbf{q}}, \mathbf{v}$, and $\dot{\mathbf{v}}$; and $\beta_{p}$ is the $l$ vector of inertia parameters [29] and assumed completely unknown in this paper.

Property 2: A suitable definition of $C(\mathbf{q}, \dot{\mathbf{q}})$ makes matrix $(\dot{D}-$ $2 C)$ skew symmetric [10].

The control objective can be specified as follows. Given a desired contact force $\lambda_{d}$ and desired trajectories $\mathbf{q}_{d}$ and $\dot{\mathbf{q}}_{d}$, which are assumed to be bounded and should satisfy the constraint equation (2), with unknown inertia parameters $\beta_{p}$, determine a control law for $\tau$ such that $\lambda, \mathbf{q}$, and $\dot{\mathbf{q}}$ asymptotically converge to $\lambda_{d}, \mathbf{q}_{d}$, and $\dot{\mathbf{q}}_{d}$.

To solve the above tracking problem, we recall that $n$ is the necessary and sufficient number of generalized coordinates required to describe the configuration of the systems. Likewise, the difference $p=n-m$ of unconstrained degrees of freedom is termed as velocity of degree of freedom. It is important to note that this $p$ denotes the number of generalized velocities, or of linear combinations thereof, that can be freely assigned without violating the kinematic constraints [24]. Henceforth, any set of linearly independent velocity variables will be termed a set of independent generalized velocities.

We thus let $\mathbf{v}$ be a $p$ dimensional vector of independent generalized velocities. Moreover, we let $R(\mathbf{q})$ be an $n \times p$ matrix defined so that it maps vector $\mathbf{v}$ into a vector of feasible generalized velocities $\dot{\mathbf{q}}$ that obeys the kinematic constraints (2). That is

$$
\dot{\mathbf{q}}=R(\mathbf{q}) \mathbf{v} \text {. }
$$

Methods for obtaining the expression of $R(\mathbf{q})$ may refer to [10] and [22]. Based on (2) and (4), it can easily be verified that

$$
R^{T}(\mathbf{q}) J^{T}(\mathbf{q})=0 .
$$

Thus, $R(\mathbf{q})$ is an orthogonal complement of $J$.

Differentiating (4), we can obtain

$$
\ddot{\mathbf{q}}=R \dot{\mathbf{v}}+\dot{R} \mathbf{v} .
$$

Therefore, the dynamic equation (1), when satisfying the nonholonomic constraint (2), can be reformulated as

$$
\begin{aligned}
\dot{\mathbf{q}} & =R(\mathbf{q}) \mathbf{v} \\
D(\mathbf{q}) R(\mathbf{q}) \dot{\mathbf{v}}+C_{1}(\mathbf{q}, \dot{\mathbf{q}}) \mathbf{v}+G(\mathbf{q}) & =B(\mathbf{q}) \tau+J^{T}(\mathbf{q}) \lambda
\end{aligned}
$$

where $C_{1}(\mathbf{q}, \dot{\mathbf{q}})=D(\mathbf{q}) \dot{R}(\mathbf{q})+C(\mathbf{q}, \dot{\mathbf{q}}) R(\mathbf{q})$.

It should be noted that the whole system consists of a new dynamic model (8) together with a purely kinematic relationship (7). As will be clear in the later development, it is this cascade structure that makes it possible to design the robust control algorithm. Regarding the general treatment of modeling issues in the nonholonomic systems, the reader may refer to [25].

\section{MAIN RESUlTS}

We now consider the tracking problem of the uncertain nonholonomic mechanical systems discussed above. In the following development, the discussions will be focused on those systems, their kinematics can be converted into the so-called chained form [26]. In fact, it has been shown that many nonholonomic systems can be converted into the chained form [2], and therefore, the systems to be discussed are not very restrictive in practical applications. Also, we only consider two independent generalized velocities $(p=2)$ cases, just for the sake of simplicity. The results can be extended to a general case. We should emphasize that our goal in this paper is to develop a force/motion tracking control strategy in a simpler setting that reveals its essential features. Interesting examples for two independent generalized velocities case include tricycle-type mobile robots, cars towing several trailers, the knife edges, and a vertical rolling wheel (see, e.g., [2]).

\section{A. Controller Design}

In the following development, it is assumed that there exists a coordinate transformation, $\mathbf{y}=\Psi(\mathbf{q})$, and a state feedback, $\mathbf{v}=\Omega_{1}(\mathbf{q}) \mathbf{u}$, so that the kinematic system (7) with $p=2$ could be locally or globally converted to the chained form [26]

$$
\begin{aligned}
& \dot{y}_{1}=u_{1} \\
& \dot{y}_{j}=u_{1} y_{j+1}, \quad(2 \leq j \leq n-1) \\
& \dot{y}_{n}=u_{2} .
\end{aligned}
$$

A necessary and sufficient condition for existence of the transformation of the kinematic system (7) with $p=2$ into this chained form (single chain) was given by [3] and [18]. For the general case (multichain case), the discussion on the existence condition of the transformation may refer to [26].

Corresponding to the above transformation, the dynamic model (8) is converted as

$$
D_{2}(\mathbf{y}) R_{2}(\mathbf{y}) \dot{\mathbf{u}}+C_{2}(\mathbf{y}, \dot{\mathbf{y}}) \mathbf{u}+G_{2}(\mathbf{y})=B_{2}(\mathbf{y}) \tau+J_{2}^{T}(\mathbf{y}) \lambda
$$

where

$$
\begin{aligned}
D_{2}(\mathbf{y}) & =\left.D(\mathbf{q})\right|_{\mathbf{q}=\Psi-1(\mathbf{y})} \\
R_{2}(\mathbf{y}) & =\left.R(\mathbf{q}) \Omega_{1}(\mathbf{q})\right|_{\mathbf{q}=\Psi-1(\mathbf{y})} \\
C_{2}(\mathbf{y}, \dot{\mathbf{y}}) & =\left.\left[D(\mathbf{q}) R(\mathbf{q}) \dot{\Omega_{1}}(\mathbf{q})+C_{1}(\mathbf{q}, \dot{\mathbf{q}}) \Omega_{1}(\mathbf{q})\right]\right|_{\mathbf{q}=\Psi-1(\mathbf{y})} \\
G_{2}(\mathbf{y}) & =\left.G(\mathbf{q})\right|_{\mathbf{q}=\Psi-1(\mathbf{y})} \\
J_{2}(\mathbf{y}) & =\left.J(\mathbf{q})\right|_{\mathbf{q}=\Psi-1(\mathbf{y})} \\
B_{2}(\mathbf{y}) & =\left.B(\mathbf{q})\right|_{\mathbf{q}=\Psi-1(\mathbf{y})} .
\end{aligned}
$$

Since the desired trajectory $\mathbf{q}_{d}$ should satisfy the constraint equation (2), therefore, there must exist a desired $\mathbf{v}_{d}$ satisfying

$$
\dot{\mathbf{q}}_{d}=R\left(\mathbf{q}_{d}\right) \mathbf{v}_{d} .
$$

Remark: It should be noted that the choice of the feasible trajectory for nonholonomic systems is strongly related to its flatness [6] or the choice of back-followable outputs, and itself is an active research topic [27]. It is beyond the scope of this paper to address this issue.

Based on the fact that the kinematic system (2) can be converted into the chained form (9), there must exist a transformation $\mathbf{y}_{d}=\Psi\left(\mathbf{q}_{d}\right)$ and a state feedback, $\mathbf{v}_{d}=\Omega_{1}\left(\mathbf{q}_{d}\right) \mathbf{u}_{d}$, such that (11) can be converted into

$$
\begin{aligned}
\dot{y}_{d 1} & =u_{d 1} \\
\dot{y}_{d j} & =u_{d 1} y_{d j+1}, \quad(2 \leq j \leq n-1) \\
\dot{y}_{d n} & =u_{d 2} .
\end{aligned}
$$

With the above transformations, the tracking problem considered in this paper can be restated as seeking a strategy for specifying a control input $\tau$ for (9) and (10), subject to the condition that parameters of the mechanical systems are not exactly known, such that $\{\lambda, \mathbf{y}, \dot{\mathbf{y}}\} \rightarrow$ $\left\{\lambda_{d}, \mathbf{y}_{d}, \dot{\mathbf{y}}_{d}\right\}$.

For the development of control law, the following assumptions are required.

Assumption (A1): The trajectories $\mathbf{y}_{d}$ and $\left(d^{i} y_{d 1} / d t^{i}\right)(1 \leq i \leq$ $n-1)$ are bounded and $\lim _{t \rightarrow \infty}$ inf $\left|u_{d 1}\right|>0$. 
Remark: As will be seen from stability analysis, Assumption (A1) on the boundedness of $\mathbf{y}_{d}$ can be relaxed to: $y_{d i}(2 \leq i \leq n)$ are bounded, $y_{d 1}$ depending on $D_{2} R_{2}, C_{2}$, and $G_{2}$. If $D_{2} R_{2}, C_{2}$, and $G_{2}$ are bounded on $y_{1}$, there is no boundedness requirement for $y_{d 1}$. This is the case in the simulation example.

Assumption (A2): The matrix $R_{2}^{T} R_{2}$ is nonsingular for all $\mathbf{y} \in \Re^{n}$ and $t \in \Re$.

Remarks: The matrix $R_{2}$ is determined by the constraint matrix $J(\mathbf{q})$ and is always a full-rank matrix. Such an assumption is generally satisfied.

With the above assumptions, the following three properties, similar to [10], can be obtained by exploiting the structure of (10).

Property 3: The generalized inertia matrix $D_{3}=R_{2}^{T} D_{2}(\mathbf{q}) R_{2}$ is symmetric and positive definite.

Property 4: If $C$ is defined as that Property 2 is verified, $\left(\dot{D}_{3}-\right.$ $\left.2 R_{2}^{T} C_{2}\right)$ is a skew-symmetric matrix.

Property 5: The dynamic structure (10) is linear, in terms of the same suitable selected set of inertia parameters as used in Property 1

$$
D_{2}(\mathbf{y}) R_{2} \dot{\mathbf{u}}+C_{2}(\mathbf{y}, \dot{\mathbf{y}}) \mathbf{u}+G_{2}(\mathbf{y})=\Phi_{1}(\mathbf{y}, \dot{\mathbf{y}}, \mathbf{u}, \dot{\mathbf{u}}) \beta_{p}
$$

where $\Phi_{1}$ is a $(n \times l)$ regressor matrix and $\beta_{p}$ is the $l$ vector of inertia parameters.

Observe that complete nonholonomic mechanical system model (9) and (10) consists of the two cascaded subsystems. As a consequence, the system's generalized velocity $\mathbf{u}$ cannot be commanded directly, as is assumed in the design of controllers at the kinematic level, and instead must be realized as the output of mechanical system dynamics (10) through $\tau$. The above properties imply that the dynamics (10) retains the mechanical system structure of the original system (1), which is fundamental for designing the robust control law. In this section, we will develop a strategy so that the subsystem (9) tracks $\mathbf{y}_{d}$ with an embedded control input [i.e., $z$ in (17)], and at the same time, the output of mechanical subsystem (10) is controlled to track this embedded control input. In turn, the tracking goal can be achieved.

To design an embedded control input so that the subsystem (10) tracks $\mathbf{y}_{d}$, in the following, we define $\mathbf{e}=\left[e_{1}, e_{2}, \ldots, e_{n}\right]^{T}=\mathbf{y}-\mathbf{y}_{d}$ and $\alpha=\left[\alpha_{1}, \alpha_{2}, \alpha_{3}, \ldots, \alpha_{n}\right]^{T}$ with

$$
\begin{aligned}
& \alpha_{1}=0 \\
& \alpha_{2}=0 \\
& \alpha_{3}=-k_{2} e_{2} u_{d 1}^{2 l-1}
\end{aligned}
$$

$$
\begin{aligned}
\alpha_{4}= & -\left(e_{2}-\alpha_{2}\right)-k_{3}\left(e_{3}-\alpha_{3}\right) u_{d 1}^{2 l-1} \\
& +\frac{1}{u_{d 1}} \sum_{i=0}^{0} \frac{\partial \alpha_{3}}{\partial u_{d 1}^{[i]}} u_{d 1}^{[i+1]}+\sum_{i=2}^{2} \frac{\partial \alpha_{3}}{\partial e_{i}} e_{i+1} \\
\vdots & \\
\alpha_{n}= & -\left(e_{n-2}-\alpha_{n-2}\right)-k_{n-1}\left(e_{n-1}-\alpha_{n-1}\right) u_{d 1}^{2 l-1} \\
& +\frac{1}{u_{d 1}} \sum_{i=0}^{n-4} \frac{\partial \alpha_{n-1}}{\partial u_{d 1}^{[i]}} u_{d 1}^{[i+1]}+\sum_{i=2}^{n-2} \frac{\partial \alpha_{n-1}}{\partial e_{i}} e_{i+1}
\end{aligned}
$$

where $l=n-2, u_{d 1}^{[i]}$ means the $i$ th derivative of $u_{d 1}$, and $k_{i}(2 \leq i \leq$ $n-1)$ are positive constants.

We suppose only that the parameter vector $\beta_{p}$ is uncertain. The robust force/motion tracking control law with the unknown inertia parameter vector $\beta_{p}$, is then defined as shown in (15)-(19) at the bottom of the page, where $\mathbf{s}$ is defined as $\mathbf{s}=\mathbf{e}-\alpha ; \Phi_{1}$ is defined in Property 5; $K_{e}$ is an $n \times n$ positive definite matrix, $\rho \in R_{+}$used in (16) is the upper bounds of unknown inertia parameter $\beta_{p}$, i.e., $\left\|\beta_{p}\right\| \leq \rho$, which is assumed known. The force term $\lambda_{c}$ is defined as

$$
\lambda_{c}=\lambda_{d}-K_{\lambda}\left(\lambda-\lambda_{d}\right)
$$

where $K_{\lambda}$ is a constant matrix of force control feedback gains.

Remarks: 1) The structure of the above controller is sketched in Fig. 1. The controller consists of two parts. In the first part, $\mathbf{z}$ represents an embedded control input, which may be viewed as an adaptive controller that ensures the desired tracking of $\mathbf{y}_{d}$ for the subsystem (9) if the mechanical dynamics described by (10) were not present. In the second part, the input $\tau$ intends to regulate $\mathbf{u}$ about the embedded control input, and therefore, attempts to provide control input necessary to make the desired tracking. The control law (15) in the second part is, in a simple fashion, related to the bounds of inertia parameters $\beta_{p}$ so that the parameter variations in the dynamics can be taken into account easily.

2) For the development of the above control law, this paper takes the common assumption that the constraint matrix $J(\mathbf{q})$ is known, i.e., there are no uncertainties for the kinematic system (2). This assumption is reasonable, since the kinematical parameters are geometric and relatively easy to measure. If this is not the case, the technique developed in [28] to deal with uncertainties in the kinematic system may be applied. However, as mentioned above, the purpose of this paper is to

$$
\begin{aligned}
B_{2} \tau & =\Phi_{1}(\mathbf{y}, \dot{\mathbf{y}}, \mathbf{z}, \dot{\mathbf{z}}) \varphi-K_{e} R_{2}(\mathbf{u}-\mathbf{z})-R_{2}\left(R_{2}^{T} R_{2}\right)^{-1} \Lambda-J_{2}^{T} \lambda_{c} \\
\varphi & =-\rho \frac{\Phi_{1}^{T} R_{2}(\mathbf{u}-\mathbf{z})}{\left\|\Phi_{1}^{T} R_{2}(\mathbf{u}-\mathbf{z})\right\|} u_{d 1}+\eta \\
\mathbf{z} & =\left[\begin{array}{c}
u_{d 2}-s_{n-1} u_{d 1}-k_{n} s_{n}+\sum_{i=0}^{n-3} \frac{\partial \alpha_{n}}{\partial u_{d 1}^{[i]}} u_{d 1}^{[i+1]}+\sum_{i=2}^{n-1} \frac{\partial \alpha_{n}}{\partial e_{i}} e_{i+1}
\end{array}\right] \\
\boldsymbol{\Lambda} & =\left[\begin{array}{l}
\Lambda_{1} \\
\Lambda_{2}
\end{array}\right]=\left[\begin{array}{c}
k_{1} s_{1}+\sum_{i=2}^{n-1} s_{i} y_{i+1}-\sum_{j=3}^{n} s_{j} \sum_{i=2}^{j-1} \frac{\partial \alpha_{j}}{\partial e_{i}} y_{i+1} \\
s_{n} \\
\dot{\eta}
\end{array}=-k_{0} \eta-k_{1} s_{1}-\sum_{i=2}^{n-1} s_{i} y_{i+1}+\sum_{j=3}^{n} s_{j} \sum_{i=2}^{j-1} \frac{\partial \alpha_{j}}{\partial e_{i}} y_{i+1}\right.
\end{aligned}
$$




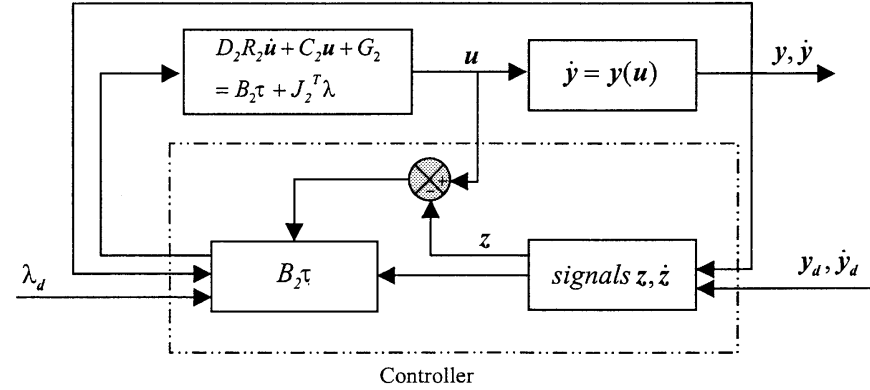

Fig. 1. Structure of the control system.

reveal the essential feature of controller development. We will not discuss this issue here for the sake of simplicity.

3) The control law (15) for the subsystem (10) is designed based on the sliding-mode theory. In this case, this sliding-mode control law is discontinuous across $\Phi_{1}^{T} R_{2}(\mathbf{u}-\mathbf{z})$, and such a control law may lead to control chattering. Chatter is undesirable in practice because it involves high control activity, and further, may excite high-frequency dynamics which were neglected in the course of modeling. This can be remedied by approximating this discontinuous control law by a continuous one inside the boundary layer. This is the commonly and practically accepted method. To do this, the term $\left(\Phi_{1}^{T} R_{2}(\mathbf{u}-\mathbf{z})\right) /\left(\left\|\Phi_{1}^{T} R_{2}(\mathbf{u}-\mathbf{z})\right\|\right)$ is replaced by $\left(\Phi_{1}^{T} R_{2}(\mathbf{u}-\mathbf{z})\right) /\left(\left\|\Phi_{1}^{T} R_{2}(\mathbf{u}-\mathbf{z})\right\|+\epsilon\right)$, where $\epsilon$ is the boundary layer thickness. This leads to tracking to within a guaranteed precision. The proof of the boundedness of the tracking error is omitted here, in order to reveal the essential feature of controller development and avoid the overwhelming mathematics.

4) Suppose $\varphi$ in control law (16) is replaced by $\hat{\beta}_{p}$, representing estimation of $\beta_{p}$, and $\hat{\beta}_{p}$ is updated by $\dot{\hat{\beta}}_{p}=-\Gamma \Phi_{1}^{T} R_{2}(\mathbf{u}-\mathbf{z})$, where $\Gamma$ is a positive definite constant matrix. With this algorithm, it can be proven that $\{\mathbf{y}, \dot{\mathbf{y}}, \lambda\} \rightarrow\left\{\mathbf{y}_{d}, \dot{\mathbf{y}}_{d}, \lambda_{d}\right\}$ as $t \rightarrow \infty$. Therefore, the proposed robust algorithm can easily be transferred into an adaptive algorithm. It is important to mention that the question of whether to use robust control or adaptive control does not have an obvious answer.

\section{B. Stability Analysis of the Closed-Loop System}

Denoting $\tilde{\mathbf{u}}=\mathbf{u}-\mathbf{z}=\left[\tilde{u}_{1} \tilde{u}_{2}\right]$, the closed-loop system of (9), (10), and (15)-(19) can be written as

$$
\begin{aligned}
\dot{s}_{1}= & \eta+\tilde{u}_{1} \\
\dot{s}_{2}= & s_{3} u_{d 1}-k_{2} s_{2} u_{d 1}^{2 l}+\left(\eta+\tilde{u}_{1}\right) y_{3} \\
\dot{s}_{3}= & s_{4} u_{d 1}-s_{2} u_{d 1}-k_{3} s_{3} u_{d 1}^{2 l}+\left(\eta+\tilde{u}_{1}\right) \\
& \cdot\left(y_{4}-\frac{\partial \alpha_{3}}{\partial e_{2}} y_{3}\right) \\
\vdots & \cdot\left(y_{n}-\sum_{i=2}^{n-2} \frac{\partial \alpha_{n-1}}{\partial e_{i}} y_{i+1}\right) \\
\dot{s}_{n-1}= & s_{n} u_{d 1}-s_{n-2} u_{d 1}-k_{n-1} s_{n-1} u_{d 1}^{2 l}+\left(\eta+\tilde{u}_{1}\right) \\
\dot{s}_{n}= & -k_{n} s_{n}-s_{n-1} u_{d 1}+\tilde{u}_{2}-\left(\eta+\tilde{u}_{1}\right) \\
& \cdot \sum_{i=2}^{n-2} \frac{\partial \alpha_{n}}{\partial e_{i}} y_{i+1} \\
D_{2} R_{2} \dot{\tilde{\mathbf{u}}=} & \Phi_{1} \varphi-\Phi_{1} \beta_{p}-K_{e} R_{2} \tilde{\mathbf{u}}-C_{2} \tilde{\mathbf{u}} \\
& -R_{2}\left(R_{2}^{T} R_{2}\right)^{-1} \Lambda-J^{T}\left(\lambda_{c}-\lambda\right)
\end{aligned}
$$

$$
\dot{\eta}=-k_{0} \eta-\Lambda_{1}
$$

Before giving the stability analysis of the above closed-loop system, the following lemmas are required.

Lemma 1: Given a differentiable function $\phi(t): R^{+} \rightarrow R$, if $\phi(t) \in$ $L_{2}$ and $\dot{\phi}(t) \in L_{\infty}$, then $\phi(t) \rightarrow 0$ as $t \rightarrow \infty$ [30].

Lemma 2: If a given differentiable function $\phi(t): R^{+} \rightarrow R$ converges to some limit value when $t \rightarrow \infty$, and if the derivative $(d \phi / d t)(t)$ of this function is the sum of two terms, one being uniformly continuous and the other one tending to zero when $t \rightarrow \infty$, then $(d \phi / d t) \rightarrow 0$ when $t \rightarrow \infty$ [17].

The stability of the above closed-loop system is established in the following theorem.

Theorem: Consider the mechanical system described by (9) and (10), subject to Assumptions (A1) and (A2), the robust adaptive controller specified by (15)-(19) ensures that all closed-loop signals are bounded. Furthermore, $y_{1}, y_{2}, \ldots, y_{n}$ asymptotically converge to $y_{d 1}$, $y_{d 2}, \ldots, y_{d n}$, and $\left(\lambda-\lambda_{d}\right)$ is inversely proportional to the norm of the matrix $\left(K_{\lambda}+I\right)$.

Proof: Multiplying $R_{2}^{T}$ on both sides of (21), using (5), one can obtain

$$
D_{3} \dot{\tilde{\mathbf{u}}}=R_{2}^{T} \Phi_{1} \varphi-R_{2}^{T} \Phi_{1} \beta_{p}-R_{2}^{T} K_{e} R_{2} \tilde{\mathbf{u}}-R_{2}^{T} C_{2} \tilde{\mathbf{u}}-\Lambda .
$$

Let us consider the positive definite function

$$
\begin{aligned}
V(t) & =V_{1}(t)+V_{2}(t) \\
V_{1}(t) & =\frac{1}{2}\left(\sum_{i=2}^{n} s_{i}^{2}+k_{1} s_{1}^{2}+\eta^{2}\right) \\
V_{2}(t) & =\frac{1}{2} \tilde{\mathbf{u}}^{T} D_{3} \tilde{\mathbf{u}} .
\end{aligned}
$$

The time derivative of $V_{1}(t)$ along the solution of (20)-(22) is

$$
\begin{aligned}
\dot{V}_{1}= & \sum_{i=2}^{n} s_{i} \dot{s}_{i}+k_{1} s_{1} \dot{s}_{1}+\eta \dot{\eta} \\
= & -\sum_{i=2}^{n-1} k_{i} s_{i}^{2} u_{d 1}^{2 l}-k_{n} s_{n}^{2}+\tilde{u}_{2} s_{n}+\left(\eta+\tilde{u}_{1}\right) \\
& \cdot\left(\sum_{i=2}^{n-1} s_{i} y_{i+1}-\sum_{j=3}^{n} s_{j} \sum_{i=2}^{j-1} \frac{\partial \alpha_{j}}{\partial e_{i}} y_{i+1}\right) \\
& +k_{1} s_{1} \eta+k_{1} s_{1} \tilde{u}_{1}-k_{0} \eta^{2}-\eta \Lambda_{1} \\
= & -\sum_{i=2}^{n-1} k_{i} s_{i}^{2} u_{d 1}^{2 l}-k_{n} s_{n}^{2}-k_{0} \eta^{2}+\tilde{\mathbf{u}}^{T} \boldsymbol{\Lambda} .
\end{aligned}
$$

The time derivative of $V_{2}(t)$ along the solution trajectory of (21) is

$$
\begin{aligned}
\dot{V}_{2}= & \tilde{\mathbf{u}}^{T}\left(R_{2}^{T} \Phi_{1} \varphi-R_{2}^{T} \Phi_{1} \beta_{p}-R_{2}^{T} K_{e} R_{2} \tilde{\mathbf{u}}\right) \\
& +\tilde{\mathbf{u}}^{T}\left(\frac{1}{2} \dot{D}_{3}-R_{2}^{T} C_{2}\right) \tilde{\mathbf{u}}-\tilde{\mathbf{u}}^{T} \boldsymbol{\Lambda} \\
= & -\tilde{\mathbf{u}}^{T} R_{2}^{T} K_{e} R_{2} \tilde{\mathbf{u}}+\tilde{\mathbf{u}}^{T} R_{2}^{T} \Phi_{1}\left(\varphi-\beta_{p}\right)-\tilde{\mathbf{u}}^{T} \boldsymbol{\Lambda}
\end{aligned}
$$

where we have used Property 4 to eliminate the term $\tilde{\mathbf{u}}^{T}\left((1 / 2) \dot{D}_{3}-\right.$ $\left.R_{2}^{T} C_{2}\right) \tilde{\mathbf{u}}$. The second term in (28) can be shown that

$$
\begin{aligned}
\left(\Phi_{1}^{T} R_{2} \tilde{\mathbf{u}}\right)^{T}\left(\varphi-\beta_{p}\right) & =\left(\Phi_{1}^{T} R_{2} \tilde{\mathbf{u}}\right)^{T}\left(-\beta_{p}-\rho \frac{\Phi_{1}^{T} R_{2} \tilde{\mathbf{u}}}{\left\|\Phi_{1}^{T} R_{2} \tilde{\mathbf{u}}\right\|}\right) \\
& \leq\left\|\Phi_{1}^{T} R_{2} \tilde{\mathbf{u}}\right\|\left(\left\|\beta_{p}\right\|-\rho\right) \leq 0
\end{aligned}
$$


from the Cauchy-Schwartz inequality and our assumption on $\left\|\beta_{p}\right\|$. Thus, one has

$$
\dot{V}_{2} \leq-\tilde{\mathbf{u}}^{T} R_{2}^{T} K_{e} R_{2} \tilde{\mathbf{u}}-\tilde{\mathbf{u}}^{T} \boldsymbol{\Lambda}
$$

Based on (27) and (30), $\dot{V}$ can be expressed as

$$
\dot{V} \leq-k_{0} \eta^{2}-k_{n} s_{n}^{2}-\sum_{i=2}^{n-1} k_{i} s_{i}^{2} u_{d 1}^{2 l}-\tilde{\mathbf{u}}^{T} R_{2}^{T} K_{e} R_{2} \tilde{\mathbf{u}} \leq 0 .
$$

The considered positive function $V(t)$ is, thus, nonincreasing. This, in turn, implies that $\eta, \mathbf{s}$, and $\tilde{\mathbf{u}}$ are bounded, and $V(t)$ converges to limit value $V_{\text {lim }}$. By the definition of $\mathbf{s}$, it concludes that $\mathbf{e}$ is bounded. Using Assumption (A1) in the theorem, it follows that $\mathbf{y}$ is bounded. In view of (20)-(22), $\dot{\mathbf{s}}, \dot{\tilde{\mathbf{u}}}$, and $\dot{\eta}$ are bounded. Thus, $\eta, \mathbf{s}$, and $\tilde{\mathbf{u}}$ are uniformly bounded. We should mention that if $D_{2} R_{2}, C_{2}$, and $G_{2}$ is bounded on $y_{1}$, there is no boundedness requirement for $y_{d 1}$. Next, we prove that $\mathbf{s}, \dot{\mathbf{s}}$, and $\eta$ tend to zero. Since $\ddot{V}$ is bounded and $\dot{V}$ is uniformly continuous, by Lemma $1, \dot{V}$ tends to zero. Therefore, $\eta$, $s_{i} u_{d 1}(2 \leq i \leq n-1), s_{n}$, and $\tilde{\mathbf{u}}$ tend to zero. By the assumption that $\lim _{t \rightarrow \infty} \inf \left|u_{d 1}\right|>0$, one concludes that $s_{i}(2 \leq i \leq n)$ tends to zero.

Differentiating $u_{d 1}^{l} \eta$ yields

$$
\begin{aligned}
\frac{d}{d t} u_{d 1}^{l} \eta=-k_{1} u_{d 1}^{l} s_{1} & +l u_{d 1}^{l-1} \dot{u}_{d 1}^{l} \eta-k_{0} u_{d 1}^{l} \eta \\
& -u_{d 1}^{l}\left(\sum_{i=2}^{n-1} s_{i} y_{i+1}-\sum_{j=3}^{n} s_{j} \sum_{i=2}^{j-1} \frac{\partial \alpha_{j}}{\partial e_{i}} y_{i+1}\right)
\end{aligned}
$$

where the first term is uniformly continuous and the other terms tend to zero. By Lemma 2, one concludes that $(d / d t)\left(u_{d 1}^{l} \eta\right)$ converges to zero, which, in turn, implies that $u_{d 1}^{l} s_{1}$ and $s_{1}$ tend to zero. Therefore, $\mathbf{s}$ and $\dot{\mathbf{s}}$ tend to zero. To complete the proof and establish asymptotic convergence of the tracking error, it is necessary to show that $\{\mathbf{y}, \dot{\mathbf{y}}\} \rightarrow\left\{\mathbf{y}_{\mathbf{d}}, \dot{\mathbf{y}}_{\mathbf{d}}\right\}$ as $t \rightarrow \infty$. This is accomplished by the following arguments.

Based on the definition of $\alpha$ given in (14) and the relationship $\mathbf{s}=$ e $-\alpha$, it is obvious that $s_{i}=0(i=1,2)$ yields $\lim _{t \rightarrow \infty} y_{i}=y_{d i}$ and $\lim _{t \rightarrow \infty} \dot{y}_{i}=\dot{y}_{d i}(i=1,2)$ because of $\alpha_{1}=\alpha_{2}=0$. From the boundedness of $u_{d 1}$, one obtains that $\alpha_{3}$ and $\dot{\alpha}_{3}$ converge to zero, which results in $\lim _{t \rightarrow \infty} y_{3}=y_{d 3}$ and $\lim _{t \rightarrow \infty} \dot{y}_{3}=\dot{y}_{d 3}$. The convergences of $\alpha_{3}$ and $\dot{\alpha}_{3}$ lead to the conclusion that $\alpha_{4}$ and $\dot{\alpha}_{4}$ converge to zero, thus, $\lim _{t \rightarrow \infty} \quad y_{4}=y_{d 4}$ and $\lim _{t \rightarrow \infty} \quad \dot{y}_{4}=\dot{y}_{d 4}$. Similarly, we can prove that $\lim _{t \rightarrow \infty} y_{i}=y_{d i}$ and $\lim _{t \rightarrow \infty} \dot{y}_{i}=\dot{y}_{d i}(5 \leq i \leq n)$. In summary, we have proved that $\{\mathbf{y}, \dot{\mathbf{y}}\} \rightarrow\left\{\mathbf{y}_{\mathbf{d}}, \dot{\mathbf{y}}_{\mathbf{d}}\right\}$ as $t \rightarrow \infty$.

To prove that $\left(\lambda-\lambda_{d}\right)$ is inversely proportional to the norm of the matrix $\left(K_{\lambda}+I\right),(21)$ can be written as

$$
\begin{aligned}
J^{T}\left(\lambda_{c}-\lambda\right)=-D_{2} R_{2} \dot{\tilde{\mathbf{u}}} & +\Phi_{1} \varphi-\Phi_{1} \beta_{p}-K_{e} R_{2} \tilde{\mathbf{u}} \\
& -R_{2}^{T} C_{2} \tilde{\mathbf{u}}-R_{2}\left(R_{2}^{T} R_{2}\right)^{-1} \Lambda=\sigma(\cdot) .
\end{aligned}
$$

It can be easily shown that $\sigma(\cdot)$ is bounded. Thus

$$
J^{T}\left(\lambda-\lambda_{d}\right)=\left(K_{\lambda}+I\right)^{-1} \sigma
$$

and therefore, $\left(\lambda-\lambda_{d}\right)$ are bounded and can be adjusted by changing the feedback gain $K_{\lambda}$. Thus, the theorem is proved.

Remarks: From the above theorem, it can be seen that $\{\mathbf{y}, \dot{\mathbf{y}}\} \rightarrow$ $\left\{\mathbf{y}_{\mathbf{d}}, \dot{\mathbf{y}}_{\mathbf{d}}\right\}$ as $t \rightarrow \infty$, which, in turn, implies that $\{\mathbf{q}, \dot{\mathbf{q}}\} \rightarrow\left\{\mathbf{q}_{\mathbf{d}}, \dot{\mathbf{q}}_{\mathbf{d}}\right\}$. On top of this, the control of contact force is also included. In the literature, there are only limited reports considering the dynamic tracking problem [10], [14], [15], and the results developed in [10], [14], and

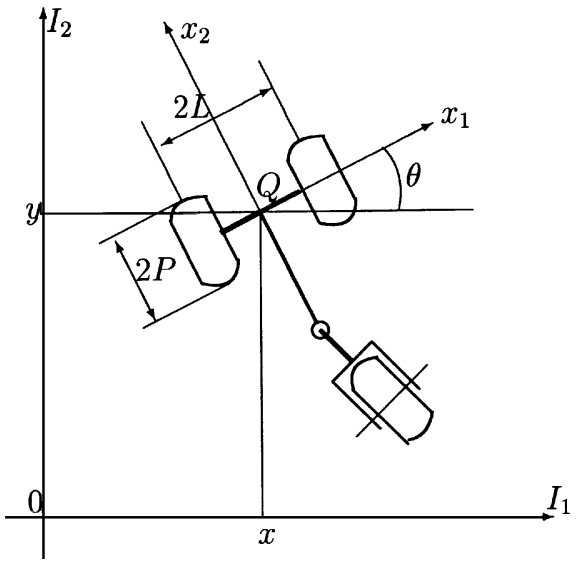

Fig. 2. Mobile robot configuration.

[15] can be considered as special cases of our result. Therefore, this paper gives a general solution for the dynamic tracking problem of the nonholonomic systems with uncertain inertia parameters.

\section{SIMULATED EXAMPLE}

A simplified model of a mobile wheeled robot moving on a horizontal plane, constituted by a rigid trolley equipped with nondeformable wheels, as shown in Fig. 2, is used to verify the validity of the control approach outlined in this paper. Details regarding the system may be found in [5].

The dynamic model can be expressed as [5]

$$
\begin{aligned}
& m \ddot{x}=\lambda \cos \theta-\frac{1}{P}\left(\tau_{1}+\tau_{2}\right) \sin \theta \\
& m \ddot{y}=\lambda \sin \theta+\frac{1}{P}\left(\tau_{1}+\tau_{2}\right) \cos \theta \\
& I_{o} \ddot{\theta}=\frac{L}{P}\left(\tau_{1}-\tau_{2}\right)
\end{aligned}
$$

where $x, y$ are the coordinates of the reference point $Q$ in the inertial frame, $\theta$ is the orientation of the basis with respect to the inertial frame, $m$ is the mass of the robot, and $I_{o}$ is its inertia moment around the vertical axis at point $Q, P$ is the radius of the wheels and $2 L$ the length of the axis of the front wheels, and $\tau_{1}$ and $\tau_{2}$ are the torques provided by motors.

The nonholonomic constraint is written as

$$
\dot{x} \cos \theta+\dot{y} \sin \theta=0 .
$$

Given the desired trajectory $\mathbf{q}_{d}=[2 \cos t, 2 \sin t, t]^{T}$, which is a circular path on the plane, and a desired contact force $\lambda_{d}=10$, the control objective is to determine a feedback control so that the trajectory $\mathbf{q}=[x, y, \theta]^{T}$ follows $\mathbf{q}_{d}$, and the contact force $\lambda_{d}$ follows $\lambda$.

The matrix $J(\mathbf{q})$ is, therefore, defined as $J(\mathbf{q})=[\cos \theta \sin \theta 0]$. The matrix $R(\mathbf{q})$ defined in (5) is chosen as

$$
R=\left[\begin{array}{cc}
-\sin \theta & 0 \\
\cos \theta & 0 \\
0 & 1
\end{array}\right]
$$

Therefore, the kinematic system (34) can be written as

$$
\begin{aligned}
& \dot{x}=-v_{1} \sin \theta \\
& \dot{y}=v_{1} \cos \theta \\
& \dot{\theta}=v_{2} .
\end{aligned}
$$


Using a diffeomorphism transformation, $\mathbf{y}=\Psi(\mathbf{q})$, and a state feedback, $\mathbf{v}=\Omega_{1}(\mathbf{q}) \mathbf{u}$, which are defined as

$$
\begin{aligned}
& y_{1}=\theta \\
& y_{2}=x \cos \theta+y \sin \theta \\
& y_{3}=-x \sin \theta+y \cos \theta \\
& u_{1}=v_{2} \\
& u_{2}=v_{1}-(x \cos \theta+y \sin \theta) v_{2}
\end{aligned}
$$

the above kinematic system is transferred into the chained form

$$
\begin{aligned}
& \dot{y}_{1}=u_{1} \\
& \dot{y}_{2}=y_{3} u_{1} \\
& \dot{y}_{3}=u_{2} .
\end{aligned}
$$

The corresponding dynamic model (33) is converted to

$$
\begin{aligned}
& {\left[\begin{array}{cc}
-m y_{2} \sin y_{1} & -m \sin y_{1} \\
m y_{2} \cos y_{1} & m \cos y_{1} \\
I_{o} & 0
\end{array}\right]\left[\begin{array}{l}
\dot{u}_{1} \\
\dot{u}_{2}
\end{array}\right]} \\
& +\left[\begin{array}{cc}
-m y_{2} \dot{y}_{1} \cos y_{1}-m \dot{y}_{2} \sin y_{1} & -m \dot{y}_{1} \cos y_{1} \\
-m y_{2} \dot{y}_{1} \sin y_{1}+m \dot{y}_{2} \cos y_{1} & -m \dot{y}_{1} \sin y_{1} \\
0
\end{array}\right]\left[\begin{array}{l}
u_{1} \\
u_{2}
\end{array}\right] \\
& =\frac{1}{P}\left[\begin{array}{cc}
-\sin y_{1} & -\sin y_{1} \\
\cos y_{1} & \cos y_{1} \\
L & -L
\end{array}\right]\left[\begin{array}{c}
\tau_{1} \\
\tau_{2}
\end{array}\right]+\left[\begin{array}{c}
\cos y_{1} \\
\sin y_{1} \\
0
\end{array}\right] \lambda
\end{aligned}
$$

with

$$
R_{2}=\left[\begin{array}{cc}
-y_{2} \sin y_{1} & -\sin y_{1} \\
y_{2} \cos y_{1} & \cos y_{1} \\
1 & 0
\end{array}\right]
$$

For the given $R(\mathbf{q})$, the desired trajectory $\mathbf{q}_{d}=[2 \cos t, 2 \sin t, t]^{T}$ satisfies $\dot{\mathbf{q}}_{d}=R\left(\mathbf{q}_{d}\right) \mathbf{v}_{d}$ with $v_{d 1}=2$ and $v_{d 2}=1$. Using the above

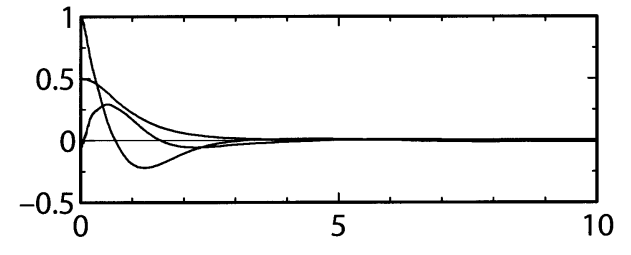

Fig. 3. Responses of $(x(t)-x(t)),\left(y(t)-y_{d}(t)\right)$, and $\left(\theta(t)-\theta_{d}(t)\right)$.

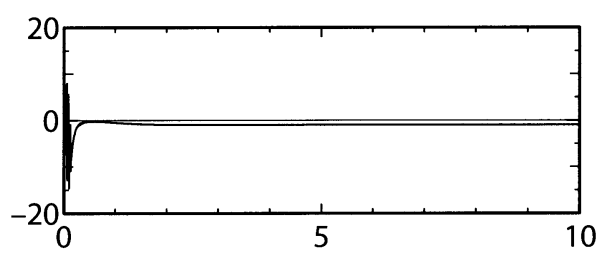

Fig. 4. Responses of $\left(\lambda_{d}-\lambda\right)$.

diffeomorphism transformation, the desired kinematic system $\dot{\mathbf{q}}_{d}=$ $R\left(\mathbf{q}_{d}\right) \mathbf{v}_{d}$ can be expressed as

$$
\begin{aligned}
& y_{d 1}=t \\
& y_{d 2}=2 \\
& y_{d 3}=0
\end{aligned}
$$

with $u_{d 1}=1$ and $u_{d 2}=0$. It is obvious that $\mathbf{y}_{d}$ and $\mathbf{u}_{d}$ satisfy Assumption (A1) regarding the desired trajectories.

The robust controller (15) is used, where the unknown parameters $\beta_{p}$ in (38) are chosen as $\beta_{p}=\left[m, I_{o}\right]^{T}$, then, the regressor matrix can be written as shown in the matrices at the bottom of the page with

$$
\left[\begin{array}{l}
e_{1} \\
e_{2} \\
e_{3}
\end{array}\right]=\left[\begin{array}{l}
y_{1}-y_{d 1} \\
y_{2}-y_{d 2} \\
y_{3}-y_{d 3}
\end{array}\right] \quad\left[\begin{array}{l}
s_{1} \\
s_{2} \\
s_{3}
\end{array}\right]=\left[\begin{array}{c}
e_{1} \\
e_{2} \\
e_{3}+k_{2} e_{2} u_{d 1}
\end{array}\right] .
$$

The physical values for this simulation are $m=0.5, I_{o}=0.5$, and $L=P=1$. The initial positions and velocities of the wheeled robot are chosen as $\mathbf{q}(0)=[3,0,0.5]$ and $\dot{\mathbf{q}}(0)=[0,0,0]$. In the simulation, the design parameters are set as $K_{e}=\operatorname{diag}(5,5), k_{0}=$ $k_{1}=k_{2}=k_{3}=5, \rho=1, K_{\lambda}=10$, and $\eta(0)=0$. In order to reduce the control chattering, the boundary layer is chosen as $\epsilon=2$.

The results of the simulation are shown in Figs. 3-5. Fig. 3 shows the trajectory tracking errors of $\left(\mathbf{q}-\mathbf{q}_{d}\right)$, Fig. 4 shows the force tracking

$$
\begin{aligned}
\Phi_{1}(\mathbf{y}, \dot{\mathbf{y}}, \mathbf{z}, \dot{\mathbf{z}}) & =\left[\begin{array}{cc}
-y_{2} \dot{z}_{1} \sin y_{1}-\dot{z}_{2} \sin y_{1}-y_{2} \dot{y}_{1} z_{1} \cos y_{1}-\dot{y}_{2} z_{1} \sin y_{1}-\dot{y}_{1} z_{2} \cos y_{1} & 0 \\
y_{2} \dot{z}_{1} \cos y_{1}+\dot{z}_{2} \cos y_{1}-y_{2} \dot{y}_{1} z_{1} \sin y_{1}+\dot{y}_{2} z_{1} \cos y_{1}-\dot{y}_{1} z_{2} \sin y_{1} & 0 \\
0 & \dot{z}_{1}
\end{array}\right] \\
\dot{\eta} & =-k_{0}-k_{1} s_{1}-y_{3} s_{2} \\
\mathbf{z} & =\left[\begin{array}{c}
u_{d 1}+\eta \\
u_{d 2}-s_{2} u_{d 1}-k_{3} s_{3}-k_{2} e_{2} u_{d 1}-k_{2} e_{3} u_{d 1}
\end{array}\right] \\
\boldsymbol{\Lambda} & =\left[\begin{array}{c}
\Lambda_{1} \\
\Lambda_{2}
\end{array}\right]=\left[\begin{array}{c}
k_{1} s_{1}+s_{2} y_{3}+k_{2} s_{3} y_{3} u_{d 1} \\
s_{3}
\end{array}\right] \\
\left(R_{2}^{T} R_{2}\right)^{-1} & =\left[\begin{array}{cc}
1 & -y_{2} \\
-y_{2} & y_{2}^{2}+1
\end{array}\right]
\end{aligned}
$$




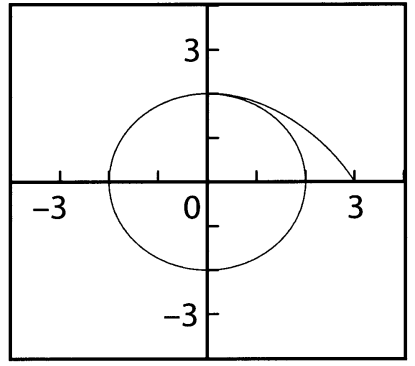

Fig. 5. Geometric trajectory of $x$ via $y$.

errors of $\left(\lambda-\lambda_{d}\right)$, and the geometric trajectory of $x$ via $y$ is shown in Fig. 5. These results confirm the validity of the proposed algorithm. In simulations, we have tested different sets of the inertia parameters using only one set of the upper bounds (which are valid for all sets of the inertia parameters) and the results are all similar, which shows the robustness of the proposed scheme. Due to space limitation, the simulation results for different sets of the inertia parameters will not be included.

\section{CONCLUSION}

In this paper, the trajectory and force tracking problem is addressed for a class of uncertain nonholonomic mechanical systems, and a robust adaptive controller is presented at the dynamic level. The controller guarantees that the configuration state of the system asymptotically tracks the desired trajectory and the force tracking error is bounded with a controllable bound. The application to a simplified mobile robot is described, and the simulation results show the effectiveness of the approach.

\section{ACKNOWLEDGMENT}

The authors wish to thank anonymous referees for the precise comments and corrections that have enabled the authors to produce the final form of this paper.

\section{REFERENCES}

[1] R. W. Brockett, "Asymptotic stability and feedback stabilization," in Differential Geometric Control Theory, R. W. Brockett, R. S. Millman, and H. J. Sussmann, Eds. Cambridge, MA: Birkhaüser, 1983.

[2] I. Kolmanovsky and N. H. McClamroch, "Developments in nonholonomic control problems," IEEE Control Syst. Mag., vol. 15, pp. 20-36, June 1995.

[3] I. Kolmanovsky, M. Reyhanoglu, and N. H. McClamroch, "Switched mode feedback control laws for nonholonomic systems in extended power form," Syst. Control Lett., vol. 27, pp. 29-36, 1996.

[4] A. M. Bloch, M. Reyhanoglu, and N. H. McClamroch, "Control and stabilization of nonholonomic dynamic systems," IEEE Trans. Automat. Contr., vol. 37, pp. 1746-1757, Nov. 1992

[5] G. Campion, B. d'Andrea-Novel, and G. Bastin, "Controllability and state feedback stabilizability of nonholonomic mechanical systems," in Advanced Robot Control, C. Canudas de Wit, Ed. New York: SpringerVerlag, 1993, pp. 106-124.

[6] M. Fliess, J. Levine, P. Martin, and P. Rouchon, "Flatness and defect of nonlinear systems: Introductory theory and examples," Int. J. Control, vol. 61, no. 6, pp. 1327-1361, 1995.
[7] A. Kapitanovsky, A. A. Goldenberg, and J. K. Mills, "Dynamic control and motion planning technique for a class of nonlinear systems with drift," Syst. Control Lett., vol. 21, pp. 363-369, 1993.

[8] R. M'Closkey and R. Murray, "Extending exponential stabilizers for nonholonomic systems from kinematic controllers to dynamic controllers," in Proc. IFAC Symp. Robot Control, 1994.

[9] B. Maschke and A. van der Schaft, "A Hamiltonian approach to stabilization of nonholonomic mechanical systems," in Proc. 33rd IEEE Conf. Decision and Control, Dec. 1994, pp. 2950-2954.

[10] C.-Y. Su and Y. Stepanenko, "Robust motion/force control of mechanical systems with classical nonholonomic constraints," IEEE Trans. Automat. Contr., vol. 39, pp. 609-614, Mar. 1994.

[11] R. M. Murray, "Nonlinear control of mechanical systems: A Lagrangian perspective," in Proc. IFAC Symp. Nonlinear Control Systems Design, 1995, pp. 349-360.

[12] R. Colbaugh, E. Barany, and K. Glass, "Adaptive control of nonholonomic robotic systems," J. Robot. Syst., vol. 15, pp. 365-393, 1998.

[13] S. S. Ge, J. Wang, T. H. Lee, and G. Y. Zhou, "Adaptive robust stabilization of dynamic nonholonomic chained systems," J. Robot. Syst., vol. 18 , pp. 119-133, 2001.

[14] W. Dong, W. L. Xu, and W. Huo, "Trajectory tracking control of dynamic nonholonomic systems with unknown dynamics," Int. J. Robust Nonlinear Contr., vol. 9, pp. 905-922, 1999.

[15] J.-M. Yang and J.-H. Kim, "Sliding-mode control for trajectory tracking of nonholonomic wheeled mobile robots," IEEE Trans. Robot. Automat., vol. 15, pp. 578-587, June 1999

[16] Ju. I. Neimark and N. A. Fufaev, Dynamics of Nonholonomic Systems. Providence, RI: Amer. Math. Soc., 1972, vol. 33, Translations of Mathematical Monographs.

[17] C. Samson, "Control of chained systems application to path following and time-varying point-stabilization of mobile robots," IEEE Trans. Automat. Contr., vol. 40, pp. 64-77, Jan. 1995.

[18] R. M. Murray and S. S. Sastry, "Nonholonomic motion planning: Steering using sinusoids," IEEE Trans. Automat. Contr, vol. 38, pp. 700-716, May 1993.

[19] Y. Stepanenko and C.-Y. Su, "Adaptive motion/force control of mechanical systems with nonholonomic Phaffian constraints," in Proc. American Control Conf., 1995, pp. 375-379.

[20] N. Sarkar, X. Yun, and V. Kumar, "Control of contact interactions with acatastatic nonholonomic constraints," Int. J. Robot. Res., vol. 16, pp. 357-374, 1997.

[21] Z.-P. Jiang and H. Nijmeijer, "A recursive technique for tracking control of nonholonomic systems in chained form," IEEE Trans. Automat. Contr., vol. 42, pp. 265-279, Feb. 1999.

[22] C.-Y. Su, Y. Stepanenko, and A. A. Goldenberg, "Reduced order model and robust control architecture for mechanical systems with nonholonomic Pfaffian constraints," IEEE Trans. Syst., Man, Cybern. A, vol. 29 pp. 307-313, May 1999.

[23] O. J. Sordalen and C. Canudas de Wit, "Exponential control law for a mobile robot: Extension to path following," in Proc. 1992 IEEE Int. Conf. Robotics and Automation, 1992, pp. 2158-2163.

[24] S. Ostrovskaya and J. Angeles, "Nonholonomic systems revisited within the framework of analytical mechanics," Appl. Mech. Rev., vol. 51, pp. 415-433, 1998.

[25] X. Yun and N. Sarkar, "Unified formulation of robotic systems with holonomic and nonholonomic constraints," IEEE Trans. Robot. Automat., vol. 14, pp. 640-650, Aug. 1998.

[26] G. Walsh and L. G. Bushnell, "Stabilization of multiple input chained form control systems," Syst. Control Lett., vol. 25, pp. 227-234, 1995.

[27] M. A. Benayad, G. Campion, V. Wertz, and M. E. Achhab, "Steering a mobile robot: Selection of a velocity profile satisfying dynamical constraints," Asian J. Contr., vol. 2, pp. 219-229, 2000.

[28] Z. P. Jiang and J.-B. Pomet, "Global stabilization of parametric chained-form systems by time-varying dynamic feedback," Int. J. Adaptive Control Signal Processing, vol. 10, pp. 47-59, 1996.

[29] M. W. Spong, "On the robust control of robot manipulators," IEEE Trans. Automat. Contr., vol. 37, pp. 1782-1786, Nov. 1992.

[30] V. M. Popov, Hyperstability of Control Systems. New York: SpringerVerlag, 1973 